\title{
Pseudo-membranous colitis
}

\author{
S. J. M. GOULSTON AND V. J. McGOVERN \\ From the A. W. Morrow Department of Gastroenterology and the Fairfax \\ Institute of Pathology, Royal Prince Alfred Hospital, Sydney, Australia
}

EDITORIAL SYNOPSIS $\mathrm{lt}$ is not generally recognized that pseudo-membranous enterocolitis may attack the colon only and has to be considered as a differential diagnosis for ulcerative colitis. Twelve cases in which the colon only was attacked are described in this paper. They fall into two groups, one group associated with primary disease of the colon, particularly with obstruction, and the other with general disease such as pneumonia or uraemia or septicaemia. It is suggested that the condition is due to a toxic agent acting locally within the mucosa and is a separate entity from ischaemic colitis or staphylococcal enterocolitis.

Mystery and controversy still surround the entity of pseudo-membranous enterocolitis. Emphasis has been placed on the condition occurring postoperatively (Pettet, Baggenstoss, Dearing, and Judd, 1954; van Prohaska, Mock, Baker, and Collins, 1961), and in association with antibiotic therapy (Cummins, 1961; Hale and Cosgriff, 1957; Pearce and Dineen, 1960). Many authors have claimed that it is always due to staphylococcal infection and is in fact a staphylococcal enterocolitis (van Prohaska, 1959). It has also been well established that it may occur without operation (Kleckner, Bargen, and Baggenstoss, 1952), but the medical picture and its importance has been less well emphasized. The fact that only the large bowel may be involved has not received much attention.

In the course of a study of the causes of acute colitis other than ulcerative colitis and granulomatous colitis causing or contributing to death in the decade 1950 to 1960,14 cases of pesudo-membranous disease were found, in all except two the disease being confined to the colon. The clinical features and pathology are described and an account is given of the order in which the histopathological events occur.

\section{CLINICAL MATERIAL}

The 14 cases were readily divided into two groups, one with a primary disease in the colon and the other in which there was a primary medical disease, usually chronic renal or cardiac insufficiency. In the first group, the precipitating factor was always a major degree of colonic obstruction, in the second it was pneumonia, uraemia, or septicaemia.

Colonic obstruction was present in five cases, in three due to adenocarcinoma of the sigmoid colon, in one to radio-necrosis in the sigmoid following irradiation to the cervix for carcinoma three years before, and one associated with a huge pseudotumour of the iliac crest obstructing the rectum in a haemophiliac. In four of five cases, the pseudomembranous colitis occurred proximal to the site of obstruction, in one instance also involving the ileum, while in the fifth case there was evidence of colitis both proximally and distally. This group included two patients under the age of 50 years. In three cases the colitis was regarded as being the cause of death and in the other two it was a contributing factor. Details are given in Table I and Figure 1.

In the group with a primary medical disease there

TABLE I

CLINICAL DETAILS OF FIVE CASES WITH COLONIC OBSTRUCTION

\begin{tabular}{|c|c|c|c|c|c|c|c|c|}
\hline \multicolumn{2}{|c|}{ Case No. } & Sex & Age & Primary Disease & Precipitating Factor & Site of Obstruction & Extent of Colitis & Cause of Death \\
\hline 1 & N.T. & $\mathbf{M}$ & 35 & Haemophilia & Pseudo-tumour & Rectum & Proximal & Yes \\
\hline 2 & E.K. & $\mathbf{F}$ & 78 & Carcinoma sigmoid & Tumour & Sigmoid & Proximal & Yes \\
\hline 3 & G.I. & $\mathbf{F}$ & 66 & Carcinoma sigmoid & Tumour & Sigmoid & Proximal and distal & Contributory \\
\hline 4 & R.C. & $\mathbf{M}$ & 84 & Carcinoma sigmoid & Tumour & Sigmoid & Proximal & Contributory \\
\hline 5 & A.M. & $F$ & 47 & Carcinoma cervix & Radio-necrosis & Sigmoid & Proximal & Yes \\
\hline
\end{tabular}




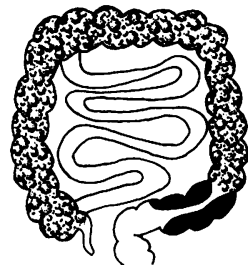

Ca.

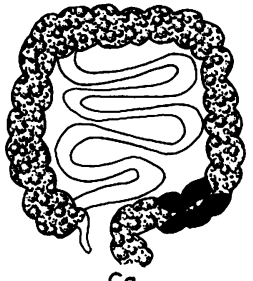

$\mathrm{Ca}$.

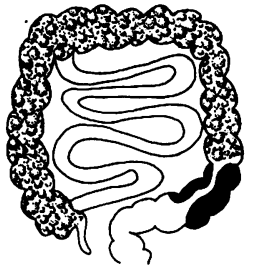

Ca.
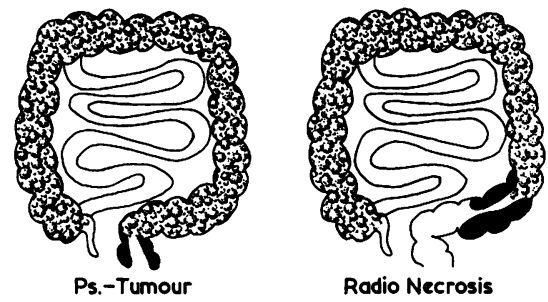

Radio Necrosis

FIG. 1. Sites of obstruction and extent of disease in five cases with colonic obstruction.

were nine cases, seven patients in the seventh decade and two aged 53 and 39 respectively. In these patients there was usually clear evidence of chronic cardio-renal disease and the colitis appeared to have occurred when precipitating factors such as uraemia or cardiac failure or pneumonia supervened. In only two cases out of nine could the colitis be regarded as causing death and in four others it was a contributing factor. Details are outlined in Table II.

The outstanding symptom in all 14 cases was diarrhoea of all grades of severity; pain was marked in only one and was altogether absent in ten. Haemorrhage was infrequent but occasionally important. The following are typical case histories.

\section{ILLUSTRATIVE CASE REPORTS}

CASE 1 N.T., a man aged 35, was a haemophiliac who had been under continuous observation since 1956. Between 1956 and 1963 he had been admitted 12 times, on many occasions for gastrointestinal bleeding. Over the latter years he developed a huge pseudo-tumour of the right iliac crest.

On 5 April 1963 he was re-admitted because of severe diarrhoea, abdominal cramps, and vomiting for six days. The abdomen was distended and tense, and plain radiographs showed gaseous distension with fluid levels. He was managed as a case of intestinal obstruction, but despite gastric suction and intravenous fluids and electrolytes, his serum electrolytes were difficult to maintain at normal levels. He became acidotic and developed peripheral vascular failure and died four days after admission.

At necropsy the gastrointestinal tract down to the caecum was relatively normal. The colon was dilated throughout its length as far as the mid-rectum and exhibited classical pseudo-membranous colitis. The rectum was markedly compressed by the pseudotumour.

CASE 3 G.I., a woman aged 69, had been treated as a diabetic for the past four years with tolbutamide. For the past 18 months she had had intermittent loose motions with rectal bleeding but had not received treatment. She had gradually deteriorated and was admitted on 25 October 1961, debilitated and vomiting. The abdomen was distended and tense. Plain radiographs of the abdomen revealed evidence of large bowel obstruction. Because of her condition she was treated conservatively and died 36 hours after admission.

At necropsy she had a fungating carcinoma completely surrounding the lumen and obstructing the proximal portion of the sigmoid colon. The remainder of the colon above this, as far as the caecum, was dilated and showed evidence of classical pseudo-membranous colitis. The rectum below the lesion showed the same pathology.

CASE 5 A.M., a woman aged 47, received radiation therapy to the lower abdomen following panhysterectomy for carcinoma of the cervix. Afterwards she had normal and regular bowel motions for two years but then began to suffer from diarrhoea, consisting of upwards of 12 loose motions daily with slime and blood. She was found

TABLE II

CLINICAL DETAILS OF NINE CASES WITH PRIMARY MEDICAL DISEASE

\begin{tabular}{|c|c|c|c|c|c|c|c|}
\hline \multicolumn{2}{|c|}{ Case No. } & Sex & Age & Primary Disease & Precipitating Factor & Extent & Cause of Death \\
\hline 6 & N.M. & $\mathbf{M}$ & 61 & Chronic renal & Uraemia & Universal & - \\
\hline 7 & C.K. & $\mathbf{F}$ & 78 & Chronic renal & Uraemia & Universal & - \\
\hline 8 & N.E. & $\mathrm{F}$ & 61 & Chronic renal & Pneumonia & Universal & Contributory \\
\hline 9 & G.I. & $\mathbf{M}$ & 67 & Chronic cardiac & Myocardial infarction & Left-sided & _ \\
\hline 10 & R.H. & $\mathbf{M}$ & 60 & Chronic cardiac & Pneumonia & Universal + ileum & Yes \\
\hline 11 & E.B. & $\mathbf{M}$ & 69 & Chronic cardiac & Pneumonia & Universal & Yes \\
\hline 12 & C.H. & $\mathbf{M}$ & 62 & $\begin{array}{l}\text { Lymphatic } \\
\text { leukaemia }\end{array}$ & Septicaemia & Universal + ileum & Contributory \\
\hline 13 & G.P. & $\mathbf{M}$ & 53 & Toxic psychosis & Pneumonia & $\begin{array}{l}\text { Transverse, descending, sigmoid } \\
\text { colon }\end{array}$ & Contributory \\
\hline 14 & J.H. & $\mathbf{M}$ & 39 & $\begin{array}{l}\text { Chronic duodenal } \\
\text { ulcer }\end{array}$ & Pneumonia & $\begin{array}{l}\text { Transverse, descending sigmoid } \\
\text { colon }\end{array}$ & Contributory \\
\hline
\end{tabular}


to have an indurated abdominal scar and a hard mass in the left iliac fossa thought to be due to a recurrence of carcinoma. Two weeks later she developed intestinal obstruction and was admitted to hospital. A caecostomy was performed but she died next day. At necropsy no residual carcinoma was found. The colon was distended from the caecum to the mid-sigmoid colon. From the centre of the sigmoid colon the bowel was markedly narrowed and partly embedded in fibrous tissue. There was considerable fibrous tissue in the pelvic colon with a thickened pelvic peritoneum and the sigmoid colon and rectum were bound together in the ischio-rectal fossa. Numerous ulcers were present in this part of the sigmoid colon and rectum. Microscopic examination disclosed radio-necrosis as the cause of the ulcers in the rectum and sigmoid colon, with pseudo-membraneous colitis involving the whole colon above the sigmoid obstruction and also two-thirds of the ileum.

\section{PATHOLOGY}

The bowel in this disorder is usually dilated and has lost its mucosal pattern. The mucosal surface has slightly raised, yellowish-greenish foci, varying in size and appearance from bran-like particles to

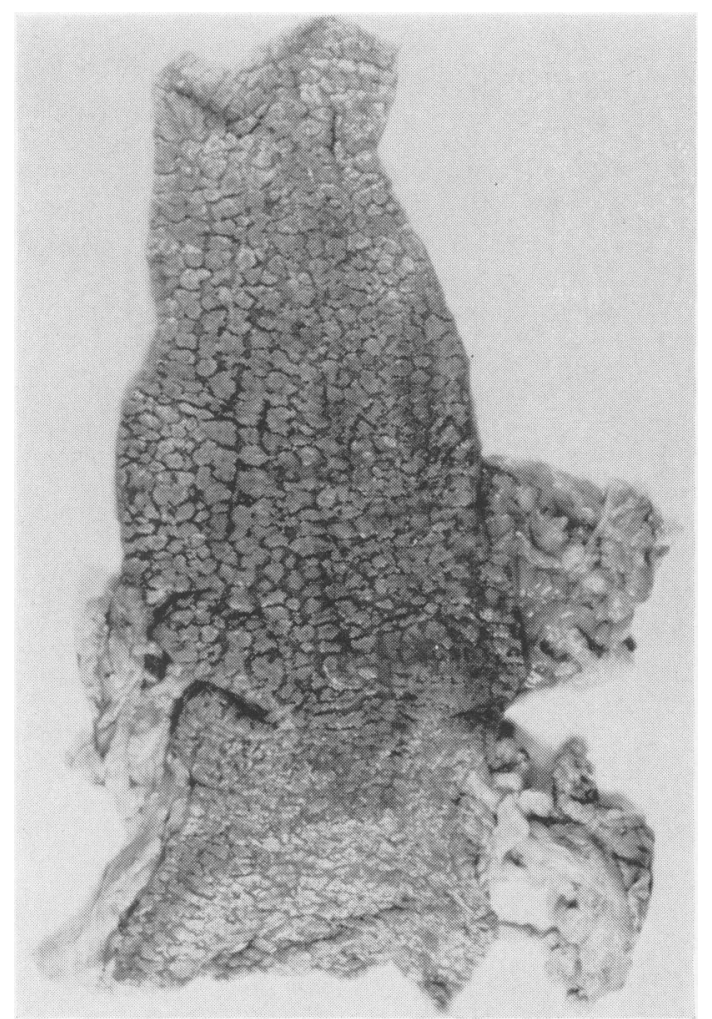

FIG. 2. A segment of colon (case 2) showing typical slightly raised plaques of exudate. large areas of yellow friable membrane which the inexperienced may interpret as ulceration because of the sharp demarcation and fibrinous surface (Figs. 2 and 3). Ulcers occur and even perforate leading to peritonitis but in most of our cases death supervened before ulceration occurred.

Microscopically, the exudate has a characteristic appearance. It is composed of fibrin, mucus, and a variable number of leucocytes. It forms in small foci by a process of fibrinoid necrosis of the surface of the mucosa and from this focus fibrinous material erupts and spreads laterally. At the same time, subjacent glandular tubules secrete excessive amounts of mucus which mixes with the exuded fibrin. Adjacent exudates, together with the necrotic superficial zones of mucosa, coalesce to form an extensive membrane. Capillaries in the mucosa may participate in the fibrinoid necrosis but vasculitis it not a feature of the lesion.

The abrupt change from normal mucosa to dilated, actively secreting glandular tubules surmounted by a layer of exudate is characteristic of the phenomenon of pseudo-membranous colitis.

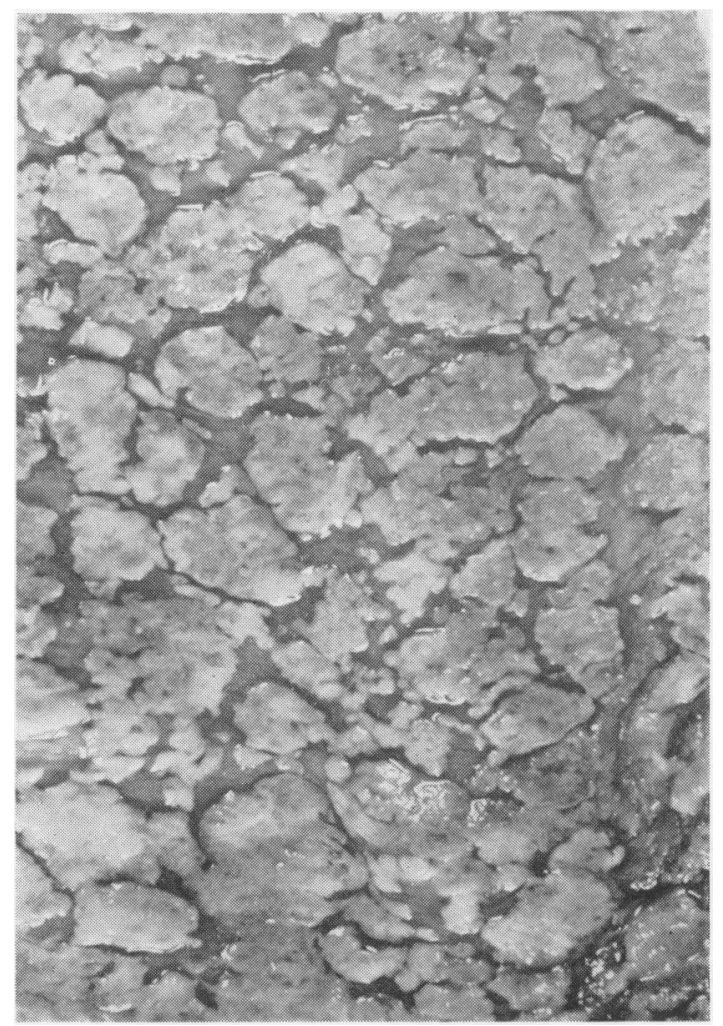

FIG. 3. A close-up view to show normal mucosa between the small membranous plaques (case 2). 


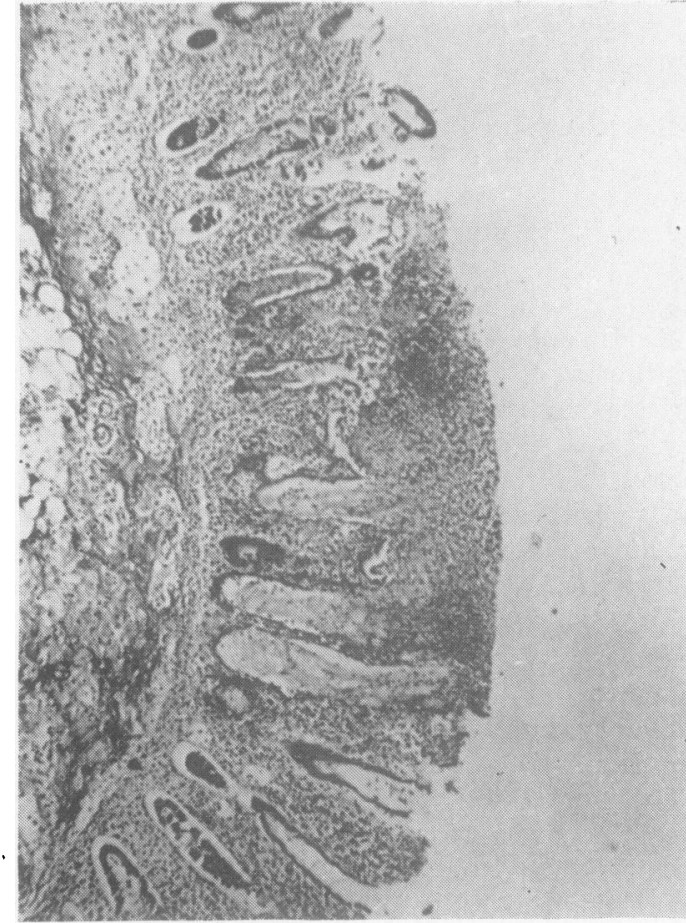

FIG. 4.

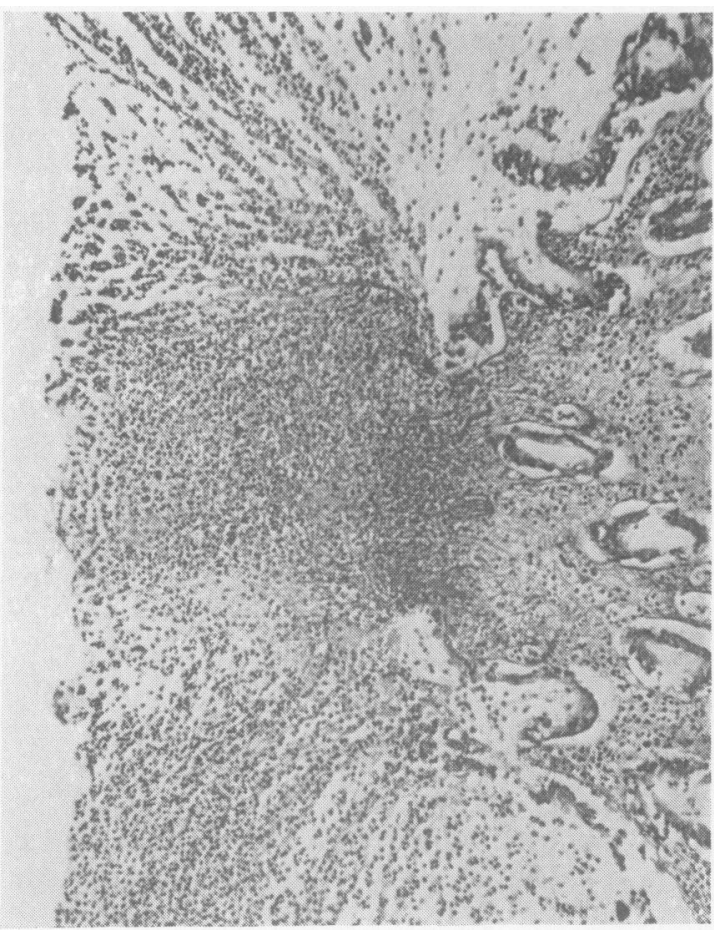

FIG. 5.

FIG. 4. A small focus of pseudo-membranous colitis in the early stages (case 14). Exudate has fused with the necrotic mucosal surface. Dilated tubules are contributing mucus to the lesion. Haematoxylin and eosin $\times 44$.

FIG. 5. The fibrinous exudate erupting from the mucosal surface and mixing with mucus on each side (case 4). Haematoxylin and eosin $\times 75$.

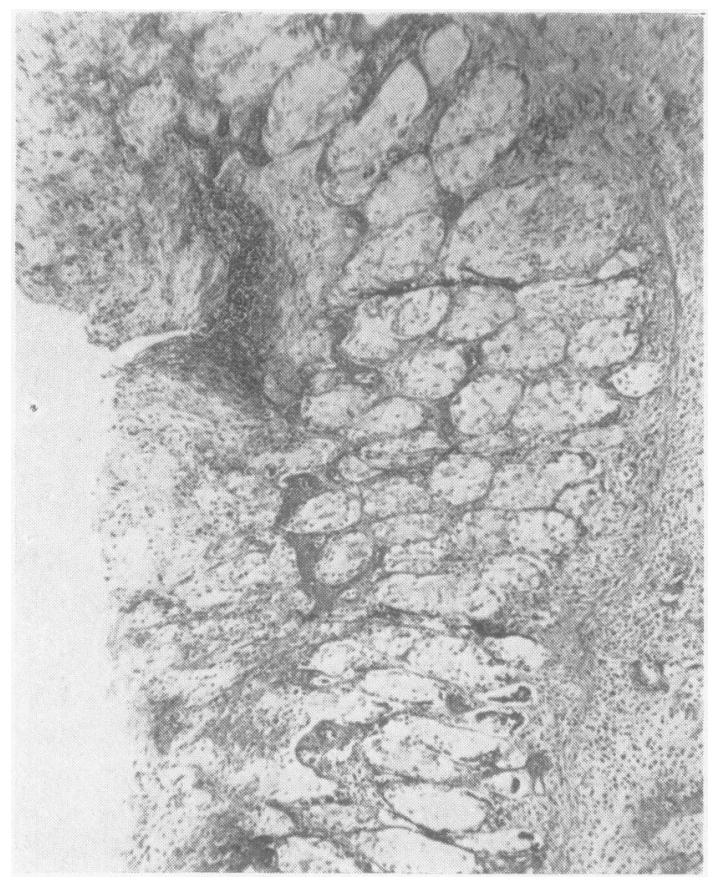

FIG. 6.

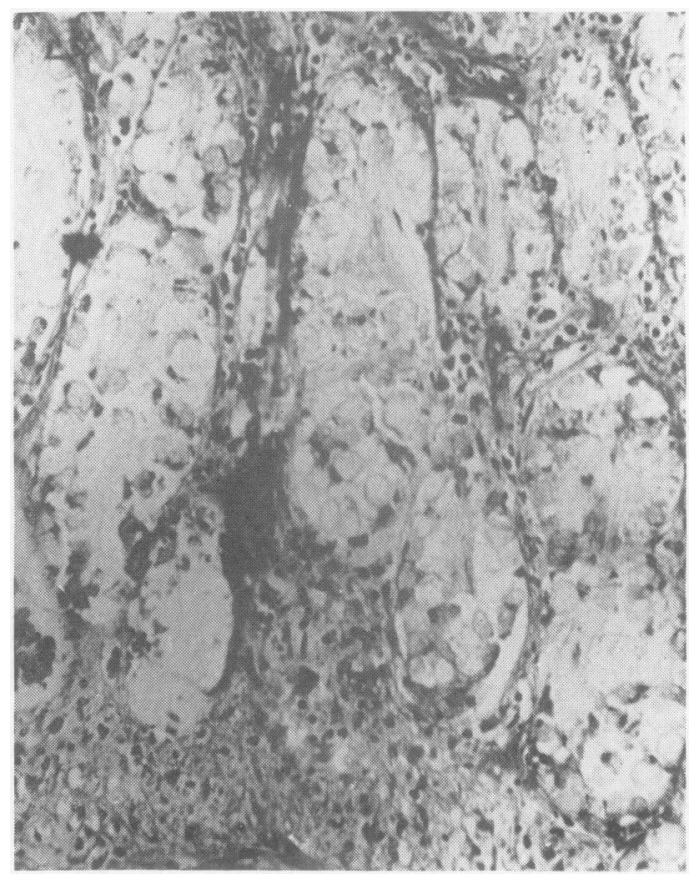

FIG. 7.

FIG. 6. Mucus from dilated mucosal tubules together with inflammatory exudate is forming a membrane on the surface of the bowel (case 5). Haematoxylin and eosin $\times 75$.

FIG. 7. A higher-power view of the tubules showing epithelial cells distended by mucus (case 5). Haematoxylin and $\operatorname{eosin} \times 150$. 


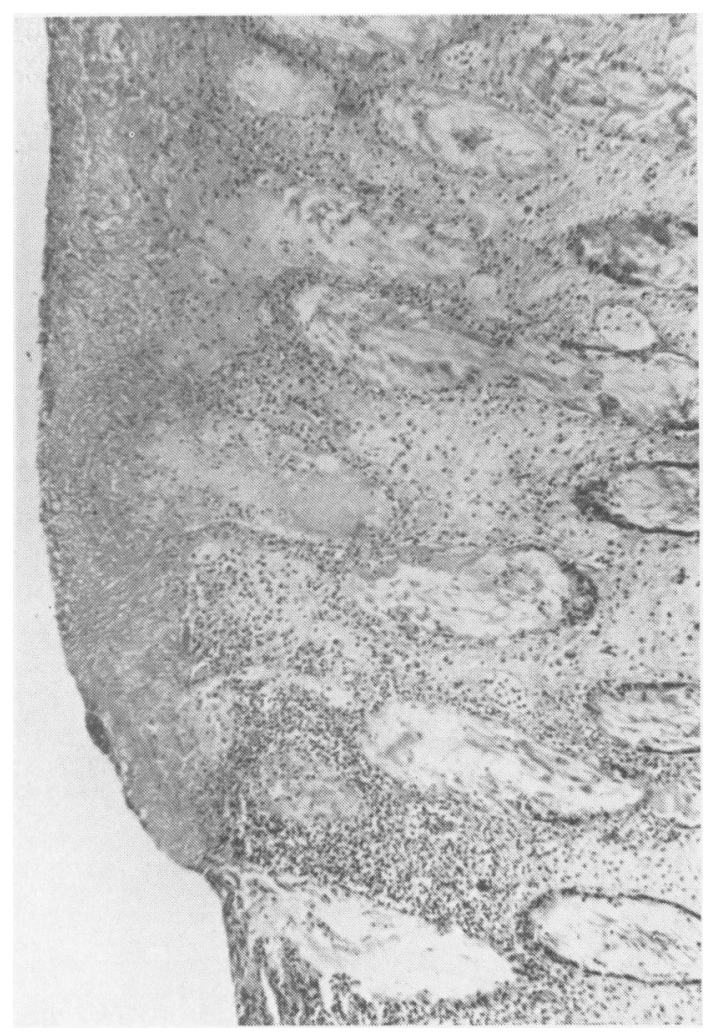

FIG. 8. The upper half of the mucosa is necrotic (case 2). Exudate and mucus have fused with it to form the membranous plaques illustrated in the figures. Haematoxylin and eosin $\times 75$.

As the disorder progresses the whole of the mucosa undergoes coagulative necrosis, and at this stage the membrane, which now consists of necrotic mucosa, mucus, fibrin, and leucocytes, may be sloughed off leaving an ulcer with a pyogenic surface which may then penetrate the muscle coat.

The submucosa is congested and oedematous and even has deposits of fibrin in it, but rarely is there much leucocytic infiltrate though phagocytes may be quite numerous (Figs. 4 to 8 ).

\section{DISCUSSION}

The pathology of this condition has produced differing opinions. Hale and Cosgriff (1957) describe a non-specific picture grossly and microscopically simulating enteritis in heavy metal poisoning, uraemia, septicaemia, bacillary dysentry, and paratyphoid fever, while Birnbaum, Laufer, and Freund (1961) state that the histological picture is identical with that found after shock, uraemia, and antibiotic or corticosteroid therapy. Reiner, Schlesinger, and
Miller (1952) likewise state that the changes are nonspecific and resemble those found in bacillary dysentry, post-operative states, septicaemia, toxic states, uraemia, mercury poisoning, and ionizing radiation. These views probably account for the differing opinions as to aetiology. We consider the pathology to be quite distinctive from other forms of colitis and would stress that the pathological entity has to be distinguished from conditions such as ulcerative colitis, granulomatous colitis, ischaemic colitis, angiitis of the large bowel, necrotizing colitis, as well as colitis due to tuberculosis, amoebiasis, and other infective agents.

Emphasis has been placed on the important aetiological factors of surgical operations and antibiotics. Pettet et al. (1954) described 94 postoperative cases, emphasizing the abrupt onset some three to five days post-operatively. It is of interest that 41 of these patients were operated upon for colonic carcinoma, and, in the light of our experience, obstruction may well have been a factor in some of these. Pearce and Dineen (1960) described 12 cases occurring five to seven days post-operatively. Reiner, Schlesinger, and Miller (1952) pointed out that pseudo-membranous colitis may follow antibiotic therapy. They describe the disease found at necropsy in five patients, four of whom had received aureomycin. However, Pettet, Baggenstoss, Judd, and Dearing (1954) reported 45 cases of pseudomembranous enterocolitis from the Mayo Clinic between 1925 and 1938 before the antibiotic era, and 49 cases from 1939 to 1952 after the introduction of antibiotics. The relative incidence was the same.

Many authors insist that the disease is in fact a staphylococcal enterocolitis (Hardaway and McKay, 1959). van Prohaska (1959) produced an experimental fatal enterocolitis in chinchillas using cultures of Staphylococcus aureus, and an enterotoxin from Staphylococcus aureus cultures from a patient with pseudo-membranous colitis; he also produced an experimental enterocolitis in chinchillas by means of antibiotics followed by an oral dose of a strain of staphylococcus. Similar experiments were carried out by Bennett, Wood, and Yardley (1956). However, staphylococcal enterocolitis is a recognized entity in its own right and the pathology is distinguishable from psuedo-membranous disease. In our cases, neither antibiotics nor operation could be said to play any part in aetiology. The important factors appeared to be chronic longstanding colonic obstruction on the one hand, and cardio-renal disease plus infection on the other. Kleckner et al. (1952) described 14 cases of pseudo-membranous enterocolitis in which the disease was not preceded by an operation. Their findings closely resembled our own. In five cases there was a colonic obstruction 
due to carcinoma of the colon, and cardiac disease was the commonest underlying lesion in the others. The most significant clinical feature was the sudden onset of profound shock due to severe watery diarrhoea. Similar findings were recorded by Bloomfield and Walters (1960) who reported five patients with this disease confined to the large bowel, not having had antecedent surgery. There was no evidence of obstruction in their cases.

Marston (1962) considers it likely that pseudomembranous enterocolitis is in fact purely ischaemic necrosis, and that it resembles the bowel in dogs which have undergone occlusion of the superior mesenteric artery. However, we have found that ischaemia produces an entirely different pathology which we have called ischaemic colitis (McGovern and Goulston, 1965). Furthermore, careful examination of liver, kidneys, and other organs in our cases failed to find evidence of damage which might have been caused by the hypotension which one would have expected to have accompanied vascular insufficiency.

Close examination of our pathological material indicates that the earliest lesion is found on the surface of the mucosa, and that it extends deeper into the mucosa and submucosa as it advances. Furthermore, the initial lesion is limited. This suggests to us that the condition is due to a toxic agent within the mucosa acting locally, and can presumably be produced as a result of colonic obstruction or in the presence of chronic cardio-renal or debilitating disease complicated by infection.

\section{REFERENCES}

Bennett, I. L., Jr., Wood, J. S., Jr., and Yardley, J. H. (1956). Staphylococcal pseudo-membranous enterocolitis in chinchillas: a clinico-pathologic study. Trans. Ass. Amer. Phycns., 69, 116-121.

Birnbaum, D., Laufer, A., and Freund, M. (1961). Pseudo-membranous enterocolitis. A clinicopathologic study. Gastroenterology, 41, 345-352.

Bloomfield, D. A., and Walters, M. N. I. (1960). Pseudo-membranous enterocolitis. Med. J. Aust., 2, 854-857.

Cummins, A. J., (1961) Pseudo-membranous enterocolitis and the pathology of nosology. Amer. J. dig. Dis., 6, 429-431.

Hale, H. W., Jr., and Cosgriff, H. J., Jr. (1957). Pseudo-membranous enterocolitis. Amer. J. Surg., 94, 710-717.

Hardaway, R. M., III, and McKay, D. G. (1959). Pseudo-membranous enterocolitis. Arch. Surg., 78, 446-457.

Kleckner, M. S., Jr., Bargen, J. A., and Baggenstoss, A. H. (1952). Pseudo-membranous enterocolitis: clinicopathologic study. Gastroenterology, 21, 212-222.

Marston, A. (1962). The bowel in shock. Lancet, 2, 365-370.

McGovern, V. J., and Goulston, A. J. M. (1965). Ischaemic enterocolitis. Gut, 6, following Paper.

Pearce, C., and Dineen, P. (1960). A study of pseudo-membranous enterocolitis. Amer. J. Surg., 99, 292-300.

Pettet, J. D., Baggenstoss, A. H., Dearing, W. H., and Judd, E. S., Jr. (1954). Postoperative pseudo-membranous enterocolitis. Surg. Gynec. Obstet., 98, 546-552.

,- Gynec. Judd, E. S., Jr., and Dearing, W. H. (1954). Generalized postoperative psuedo-membranous enterocolitis. Mayo Clin. Proc., 29, 342-349.

Prohaska, J. van, (1959). Pseudo-membranous enterocolitis. Arch. Surg., 79, 197-206.

—, Mock, F., Baker, W., and Collins, R. (1961). Pseudomembranous (staphylococcal) enterocolitis. Int. Abstr. Surg., 112, 103-115.

Reiner, L., Schlesinger, M. J., and Miller, G. M. (1952). Pseudomembranous colitis following aureomycin and chloramphenicol Arch. Path., 54, 39-67. 